\title{
A Genome-Wide Association Study of Bisphosphonate-Associated Atypical Femoral Fracture
}

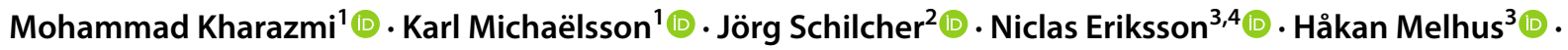 \\ Mia Wadelius ${ }^{3}$ (1) Pär Hallberg ${ }^{3}$ (1)
}

Received: 8 January 2019 / Accepted: 8 April 2019 / Published online: 20 April 2019

(c) The Author(s) 2019

\begin{abstract}
Atypical femoral fracture is a well-documented adverse reaction to bisphosphonates. It is strongly related to duration of bisphosphonate use, and the risk declines rapidly after drug withdrawal. The mechanism behind bisphosphonate-associated atypical femoral fracture is unclear, but a genetic predisposition has been suggested. With the aim to identify common genetic variants that could be used for preemptive genetic testing, we performed a genome-wide association study. Cases were recruited mainly through reports of adverse drug reactions sent to the Swedish Medical Products Agency on a nationwide basis. We compared atypical femoral fracture cases $(n=51)$ with population-based controls $(n=4891)$, and to reduce the possibility of confounding by indication, we also compared with bisphosphonate-treated controls without a current diagnosis of cancer $(n=324)$. The total number of single-nucleotide polymorphisms after imputation was $7,585,874$. A genome-wide significance threshold of $p<5 \times 10^{-8}$ was used to correct for multiple testing. In addition, we performed candidate gene analyses for a panel of 29 genes previously implicated in atypical femoral fractures (significance threshold of $p<5.7 \times 10^{-6}$ ). Compared with population controls, bisphosphonate-associated atypical femoral fracture was associated with four isolated, uncommon single-nucleotide polymorphisms. When cases were compared with bisphosphonate-treated controls, no statistically significant genome-wide association remained. We conclude that the detected associations were either false positives or related to the underlying disease, i.e., treatment indication. Furthermore, there was no significant association with single-nucleotide polymorphisms in the 29 candidate genes. In conclusion, this study found no evidence of a common genetic predisposition for bisphosphonate-associated atypical femoral fracture. Further studies of larger sample size to identify possible weakly associated genetic traits, as well as whole exome or whole-genome sequencing studies to identify possible rare genetic variation conferring a risk are warranted.
\end{abstract}

Keywords Genome-wide association study · Atypical fractures · Bisphosphonate $\cdot$ Drug-related side effects and adverse reactions $\cdot$ Pharmacogenetics

Electronic supplementary material The online version of this article (https://doi.org/10.1007/s00223-019-00546-9) contains supplementary material, which is available to authorized users.

Mohammad Kharazmi

kharazmi.mohammad@gmail.com

1 Department of Surgical Sciences, Uppsala University, Uppsala, Sweden

2 Department of Clinical and Experimental Medicine, Faculty of Health Sciences, Linköping University, Linköping, Sweden

3 Department of Medical Sciences, Uppsala University, Uppsala, Sweden

4 Uppsala Clinical Research Center, Uppsala University, Uppsala, Sweden

\section{Introduction}

For over a decade, atypical fracture of the femoral bone (AFF) has been a well-documented adverse drug reaction (ADR) associated with long-term bisphosphonate use [1]. AFF is normally preceded by weeks or months of thigh pain and is in contrast to ordinary fragility fractures related to no or minimal trauma [2]. The term 'atypical' refers to the deviant transverse pattern of the fracture-line revealed on plain radiographs of the affected femur [2]. Although not all AFFs occur after bisphosphonate exposure, there is a strong correlation with duration of bisphosphonate use. A more than 100 -fold increase in risk is seen after $4-5$ years 
of bisphosphonate use, and the risk declines rapidly after cessation of treatment [3-5].

By now, clinicians, the scientific community and patients have come to realize the many challenges associated with AFFs. Over the last decade, a 50\% decrease in prescriptions of bisphosphonates for primary and secondary prevention of fragility fractures has been seen [6]. This significant decline in preventive medication is believed to be due to fear of ADRs.

A major challenge in the prevention of AFF is the overall lack of knowledge about the mechanism behind this fracture type. Theories highlight long-term buildup of micro-cracks in the bone due to an over-suppression of bone remodeling that eventually leads up to failing skeletal integrity and stress fractures [7]. Predisposing risk factors are long-term use of bisphosphonates [3], female sex [3, 8], Asian ethnicity [9], and bowing of the femur [10]. Since only a minority of bisphosphonate users develop AFF, pathophysiological theories include a predisposing genetic trait, altered collagen cross-linking, accumulation of microdamage, increased mineralization, reduced heterogeneity of mineralization, variation in rates of bone turnover, and reduced vascularity [2].

A recent systematic review found six published studies that investigated the role of genetics on AFF in a total of 44 patients [11]. The review also identified 23 cases of AFF associated with seven different monogenetic bone disorders, of which seven cases had been exposed to a bisphosphonate. There is thus some evidence of rare genetic susceptibility loci for bisphosphonate-associated AFF. If common risk variants, i.e., genetic variants occurring among at least $1 \%$, also exist, as has been shown for many rare adverse drug reactions [12], it might be feasible to predict patients at risk through preemptive genotyping. We performed the largest case-control GWAS to date, to determine whether common genetic variants contribute to risk of bisphosphonate-associated AFF. We also performed candidate gene analyses of 29 genes that have been implicated in AFF [11].

\section{Materials and Methods}

\section{Sample Description}

The basis for case recruitment was through nation-wide spontaneous ADR reports sent from healthcare professionals to the Swedish Medical Products Agency between the years 2006 and 2015. Each patient should be at least 18 years of age and able to give informed consent. Case definition for AFF was according to the American Society for Bone and Mineral Research [2].
We collected clinical data (demographics, medical history, drug treatment history, X-ray images, and ancestry) through interviews using a standardized questionnaire, and by obtaining and reviewing medical records. Prior to genetic analysis, each case including radiographs was evaluated by at least one senior consultant in orthopedics.

Overall, 71 reported cases were available. Of these, 18 cases were not possible to include (five were deceased, five could not be reached, four declined to participate, two were not suitable to be contacted according to the reporting physician, one was not able to perform the interview, and in one case the reporting physician could not be reached). Of the remaining 53 cases, two did not pass radiograph adjudication (ordinary fragility fractures) and therefore 51 cases, all with complete fractures, were included in the study. We compared the cases with two sets of controls. In the main analysis, we utilized 4891 population controls from the Swedish Twin Registry [13], all non-related individuals. The proportion of women in this population was $46 \%$, and birth years ranged from 1911 to 1958 (1911-1919, $0.78 \%$; 1920-1929, 10.3\%; 1930-1939, 27.7\%; 1940-1949, $45.7 \% ; 1950-1958,15.5 \%)$. Information on diseases and drug treatments for controls was available by linkage to individual data from the Swedish National Patient Register and the Swedish Prescribed Drug Register. Complete linkage is enabled by use of the individual personal registration number provided to all Swedish citizens. To determine whether any positive GWAS findings might be due to confounding by indication, we also defined a matched control group, consisting of patients who had collected at least one prescription of a bisphosphonate and who did not have a current cancer diagnosis. This gave a total of 324 controls that had been prescribed bisphosphonates and thus resembling the same source population of individuals as the cases, i.e., bisphosphonate users. Four out of five matched controls were women, which corresponds well with the overall proportion of women/men prescribed bisphosphonates in Sweden according to the Swedish Prescribed Drug Register. None of the cases with AFF had a current diagnosis of cancer.

\section{Genome-Wide Array Data and Analyses}

DNA was extracted from peripheral venous blood. Cases were genotyped with the Illumina Infinium OmniExpressExome $1 \mathrm{M}$ array, and controls were genotyped with the Illumina HumanOmniExpress $700 \mathrm{~K}$ array. Genotype calls were generated using the Genome Studio software from Illumina and the Genome Reference Consortium human assembly GRCh 37 .

Genotyping quality control (QC) and data management was performed using PLINK v1.9 [14]. The resulting merged 
data included 604,238 SNPs post QC. Imputation was performed using the Sanger imputation server [15]. The pipeline with Eagle2 (v2.0.5) prephasing [16] and PBWT imputation [17] was used with the haplotype reference consortium panel as reference (v1.1) [15]. The total number of SNPs after imputation and QC was 7,585,874. All cases and controls were within the European cluster according to genetic principal component analysis (PCA), except for one case of Chilenean origin (Supplemental Fig. 1). Additional details on QC, PCA and imputation can be found in the Supplement.

Logistic regression on a genome-wide level was performed using PLINK v1.9 [14]. All genome-wide analyses were adjusted for the first four principal components. SNP effects were modeled only as additive and the conventional genome-wide significance threshold $p<5 \times 10^{-8}$ was used to correct for multiple testing [18]. Results are presented as Manhattan plots. QQ-plots are presented in Supplemental Figs. 2 and 3.

\section{Candidate Gene Analyses}

In addition to genome-wide analyses, we performed candidate gene analyses in the imputed data set for a panel of 29 genes that have been implicated in AFF (Table 1) [11]. We examined a panel consisting of 8709 SNPs distributed in these genes. We both tested all 51 cases vs all 4891 controls and all 51 cases vs the 324 matched controls. Adjustment for multiple testing was done with Bonferroni correction $\left(0.05 / 8709 \approx 5.74 \times 10^{-6}\right)$.

\section{Power Calculation}

Given a genome-wide significance level of $p<5 \times 10^{-8}$ and using an additive genetic model, our sample size was powered to detect common genetic variants with effect sizes of clinical utility [19]. We had approximately $80 \%$ power to detect an odds ratio (OR) of 3-4 for variants with a minor allele frequency (MAF) of $40 \%$, and $80 \%$ power to detect an OR of 4-5 for variants with a MAF of 20\% (Supplemental Figs. 4 and 5). Given the significance level of $p<5.74 \times 10^{-6}$ in the candidate gene analyses, we had $80 \%$ power to detect an OR of about 3 for variants with a MAF of $40 \%$, and $80 \%$ power to detect an OR of about 4 for variants with a MAF of 20\% (Supplemental Figs. 6 and 7).

\section{Results}

Characteristics of the 51 cases ( 48 women and 3 men) of bisphosphonate-associated AFF and the 324 matched controls are shown in Table 2. Most of the cases were of Swedish ethnicity $(n=47)$, while one each was of Finnish, Norwegian, British or Chilean origin.
Table 1 Candidate genes tested in the study

\begin{tabular}{|c|c|c|c|}
\hline Gene & Chromosome & Start position & End position \\
\hline ACKR3 (CXCR7) & 2 & 237476430 & 237491001 \\
\hline ACOXL & 2 & 111490150 & 111875799 \\
\hline ALPL & 1 & 21835858 & 21904905 \\
\hline CCDC147 & 10 & 106113522 & 106214848 \\
\hline CNGB1 & 16 & 57917503 & 58005020 \\
\hline COL1A2 & 7 & 94023873 & 94060544 \\
\hline CRYBB2 & 22 & 25615489 & 25627836 \\
\hline CTSK & 1 & 150768684 & 150780799 \\
\hline CYP1A1 & 15 & 75011883 & 75017951 \\
\hline DOCK2 & 5 & 169064251 & 169510386 \\
\hline EDC3 & 15 & 74922899 & 74988633 \\
\hline FN1 & 2 & 216225163 & 216300895 \\
\hline FOXK2 & 17 & 80477589 & 80602538 \\
\hline GGA3 & 17 & 73232694 & 73258444 \\
\hline GGPS1 & 1 & 235490665 & 235507847 \\
\hline HHAT & 1 & 210501596 & 210849638 \\
\hline LIPN & 10 & 90521163 & 90537999 \\
\hline MVD & 16 & 88718343 & 88729569 \\
\hline NAT8B & 2 & 73927636 & 73928467 \\
\hline NGEF & 2 & 233743396 & 233877982 \\
\hline OR2L13 & 1 & 248100493 & 248264224 \\
\hline OR51T1 & 11 & 4903049 & 4904113 \\
\hline PCK2 & 14 & 24563262 & 24579807 \\
\hline PPEF2 & 4 & 76781020 & 76823724 \\
\hline SF3B3 & 16 & 70557691 & 70608820 \\
\hline SLC15A5 & 12 & 16341419 & 16430619 \\
\hline SLC2A6 & 9 & 136336217 & 136344259 \\
\hline SYDE2 & 1 & 85622556 & 85666729 \\
\hline SYTL2 & 11 & 85405267 & 85522184 \\
\hline
\end{tabular}

Genes implicated in atypical femoral fractures [11]

\section{Genome-Wide Association Analyses-Cases Versus All Population Controls}

Bisphosphonate-associated AFF was significantly associated with four isolated single nucleotide polymorphisms (SNP) (Fig. 1a; Table 3). The first SNP was rs7729897, which is located in an intergenic region upstream of the $\mathrm{NR} 3 \mathrm{C} 1$ gene (nuclear receptor subfamily 3 group $\mathrm{C}$ member 1) on chromosome 5, OR 10.27 [95\% confidence interval (CI) $4.95,21.31] p=4.00 \times 10^{-10}$. The NR3C1 gene encodes a glucocorticoid receptor, which functions as a transcription factor that activates glucocorticoid responsive genes, and as a regulator of other transcription factors [20]. Variants of this gene have been associated with decreased bone mineral density in patients with endogenous hypercortisolism [21, 22]. 
The second SNP was rs11465606 positioned in an intron within the IL18R1 gene (interleukin 18 receptor 1) on chromosome 2, OR 6.15 [95\% CI 3.32, 11.37], $p=7.13 \times 10^{-9}$. The third SNP was rs145787127, which is located in an intron of the NTN1 (netrin 1) gene on chromosome 17, OR 7.37 [95\% CI 3.63, 14.93], $p=3.08 \times 10^{-8}$. Genetic variation within NTN1 has been linked to osteoporosis [23]. The last SNP was rs144094653, located close to the pseudogene TUBB8P5 (tubulin beta 8 class VIII pseudogene 5 on chromosome 12, OR 7.68 [95\% CI 3.70, 15.91], $p=4.20 \times 10^{-8}$.

\section{Genome-Wide Association Analyses-Cases Versus Controls with Bisphosphonate Use}

No statistically significant association with gene status was revealed when cases of bisphosphonate-associated AFF were compared with matched controls (Fig. 1b; Table 4).

\section{Candidate Gene Analyses-Cases Versus All Population Controls}

When cases of bisphosphonate-associated AFF were compared with all population controls, there were no statistically
Fig. 1 a Manhattan plot of the genome-wide association analysiscases vs all controls. b Manhattan plot of the genome-wide association analysis-cases vs matched controls. Analyses of 51 cases of bisphosphonate-associated atypical femoral fractures versus a all 4891 population controls, and b 324 matched controls. There were $7,585,874$ SNPs after imputation, and adjustment was made for genetic principal components $1-4$. The red line shows the threshold for genome-wide significance of $5 \times 10^{-8}$. a Four SNPs were statistically significant when cases were compared with all 4891 controls. The top SNP was rs 7729897 , located in an intergenic region upstream of the $\mathrm{NR} 3 \mathrm{C} 1$ gene (nuclear receptor subfamily 3 group $\mathrm{C}$ member 1) on chromosome 5, odds ratio (OR) 10.27 [95\% confidence interval $(\mathrm{CI}) 4.95,21.31] p=4.00 \times 10^{-10}$. There was also a significant association with rs11465606 positioned in an intronic region within the IL18R1 gene (interleukin 18 receptor 1) on chromosome 2, OR 6.15 [95\% CI 3.32, 11.37], $p=7.13 \times 10^{-9}$. A third significant association was with rs145787127, which is located in an intron region of the NTN1 (netrin 1) gene on chromosome 17, OR 7.37 [95\% CI 3.63, 14.93], $p=3.08 \times 10^{-8}$. The fourth significant association was with rs144094653, located close to the pseudogene TUBB8P5 (tubulin beta 8 class VIII pseudogene 5 on chromosome 12, OR 7.68 [95\% CI $3.70,15.91], p=4.20 \times 10^{-8}$. SNP single nucleotide polymorphism. b There were no statistically significant findings when cases were compared with matched controls. SNP single nucleotide polymorphism

significant associations (Fig. 2a; Table 5; Supplemental Table 1).
Table 2 Characteristics of cases of bisphosphonate-associated atypical femoral fractures and matched controls

\begin{tabular}{lll}
\hline & AFF $(n=51)$ & $\begin{array}{l}\text { Matched } \\
\text { controls } \\
(n=324)\end{array}$ \\
\hline Gender ( $n$ female, [proportion female]) & & $257[0.79]$ \\
Age $^{\mathrm{a}}$ (mean, years [range]) & $48[0.94]$ & $71.5[52-93]$ \\
PPI ( $n$, [proportion]) & $70.7[47-86]$ & $100[0.31]$ \\
Systemic corticosteroids ( $n$, [proportion]) & $17[0.33]$ & $123[0.38]$ \\
Alendronic acid $(n$, [proportion]) & $17[0.33]$ & $264[0.81]$ \\
Zoledronic acid $(n$, [proportion]) & $47[0.92]$ & $4[0.012]$ \\
Risedronic acid $(n$, [proportion]) & $2[0.039]$ & $51[0.16]$ \\
Etidronic acid $(n$, [proportion]) & $4[0.078]$ & $7[0.022]$ \\
Ibandronic acid (proportion) & $0[0]$ & $1[0.0031]$ \\
Clodronate (proportion) & $0[0]$ & $0[0]$ \\
Oral administration (proportion) & $0[0]$ & $320[0.99]$ \\
Indication for treatment with bisphosphonate & $49[0.96]$ & Unknown \\
$\quad$ Osteoporosis $(n)$ & & \\
Prophylaxis due to corticosteroid treatment $(n)$ & 45 & N/A \\
Unknown $(n)$ & 2 & \\
Fracture location & 4 & \\
Femur $(n)$ & 51 & \\
\hline
\end{tabular}

Matched controls were individuals who had collected at least one prescription of a bisphosphonate. We excluded as matched controls those individuals who had a diagnosis of cancer (any type) 12 months prior to or following first collection of a prescription of a bisphosphonate. Note that some patients have received more than one bisphosphonate

${ }^{a}$ Age at time of onset of AFF for cases, and time of first recorded collection of a prescription of a bisphosphonate for controls

$A F F$ atypical femoral fractures 


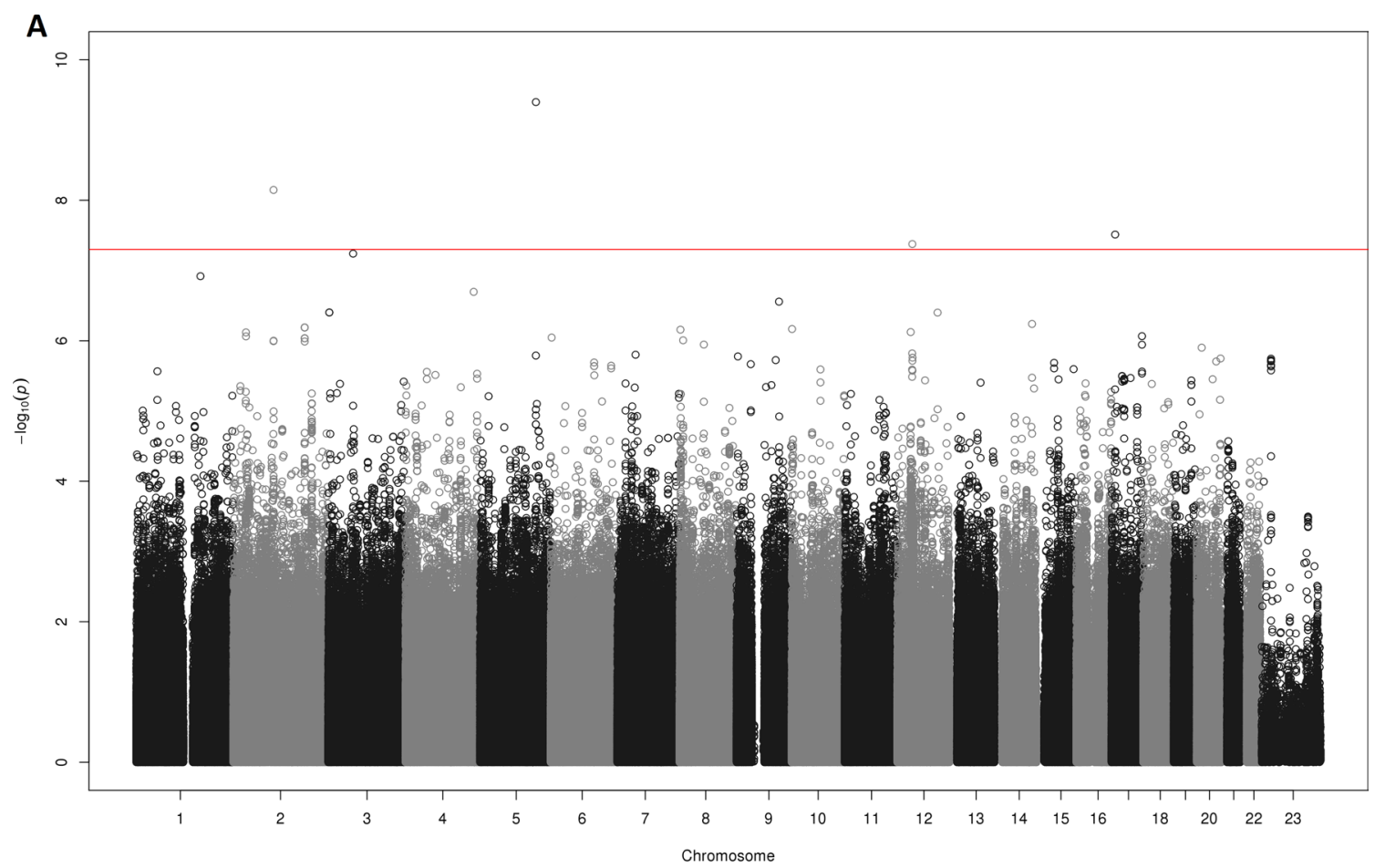

B

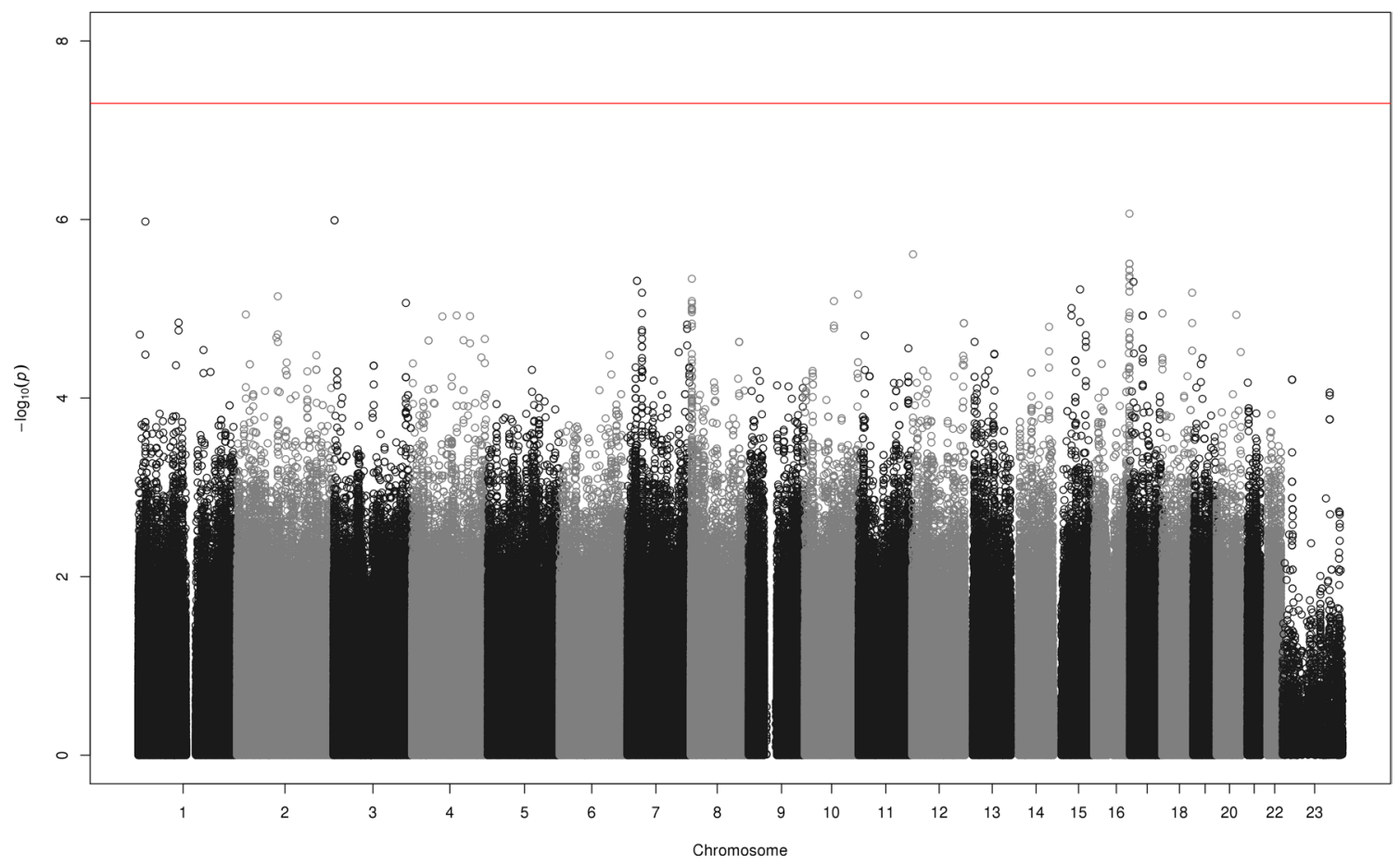


Table 3 Top genome-wide associations with bisphosphonate-associated atypical femoral fractures

\begin{tabular}{|c|c|c|c|c|c|c|c|c|c|c|c|c|}
\hline CHR & SNP & $\mathrm{BP}$ & Minor allele & $\mathrm{N}$ & OR & L95 & U95 & $p$ & GTPS & MAF cases & MAF controls & Gene \\
\hline 5 & rs7729897 & 142970862 & $\mathrm{G}$ & 4942 & 10.27 & 4.949 & 21.31 & $4.000 \times 10^{-10}$ & $\mathrm{G} / \mathrm{C}$ & 0.098 & 0.01 & \\
\hline 2 & rs11465606 & 102988300 & $\mathrm{~A}$ & 4942 & 6.149 & 3.324 & 11.37 & $7.131 \times 10^{-9}$ & $\mathrm{~A} / \mathrm{C}$ & 0.128 & 0.024 & IL18R1 \\
\hline 17 & rs 145787127 & 9142414 & A & 4942 & 7.366 & 3.633 & 14.93 & $3.076 \times 10^{-8}$ & $\mathrm{~A} / \mathrm{G}$ & 0.098 & 0.016 & NTN1 \\
\hline 12 & rs144094653 & 38593619 & A & 4942 & 7.675 & 3.704 & 15.91 & $4.201 \times 10^{-8}$ & $\mathrm{~A} / \mathrm{G}$ & 0.088 & 0.014 & \\
\hline 3 & rs73111385 & 63645410 & G & 4942 & 5.042 & 2.811 & 9.045 & $5.755 \times 10^{-8}$ & G/A & 0.137 & 0.031 & SNTN \\
\hline 1 & rs113093597 & 165017843 & A & 4942 & 6.144 & 3.137 & 12.03 & $1.205 \times 10^{-7}$ & $\mathrm{~A} / \mathrm{G}$ & 0.098 & 0.017 & \\
\hline 4 & rs191328328 & 174611710 & $\mathrm{C}$ & 4942 & 8.951 & 3.917 & 20.46 & $2.013 \times 10^{-7}$ & $\mathrm{C} / \mathrm{T}$ & 0.069 & 0.009 & \\
\hline 9 & rs 12336042 & 108538200 & A & 4942 & 6.731 & 3.252 & 13.93 & $2.774 \times 10^{-7}$ & $\mathrm{~A} / \mathrm{T}$ & 0.088 & 0.015 & TMEM38B \\
\hline 3 & rs76646538 & 2694727 & $\mathrm{C}$ & 4942 & 3.933 & 2.317 & 6.676 & $3.950 \times 10^{-7}$ & $\mathrm{C} / \mathrm{T}$ & 0.157 & 0.04 & CNTN4 \\
\hline 3 & rs6768500 & 2693258 & $\mathrm{C}$ & 4942 & 3.932 & 2.316 & 6.675 & $3.962 \times 10^{-7}$ & $\mathrm{C} / \mathrm{G}$ & 0.157 & 0.04 & CNTN4 \\
\hline 12 & rs147502517 & 103265420 & $\mathrm{~T}$ & 4942 & 7.191 & 3.354 & 15.42 & $3.972 \times 10^{-7}$ & $\mathrm{~T} / \mathrm{G}$ & 0.078 & 0.012 & PAH \\
\hline 14 & rs72698961 & 96278663 & G & 4942 & 5.128 & 2.701 & 9.734 & $5.762 \times 10^{-7}$ & $\mathrm{G} / \mathrm{A}$ & 0.118 & 0.027 & \\
\hline 2 & rs74476239 & 182754649 & $\mathrm{C}$ & 4942 & 6.925 & 3.232 & 14.84 & $6.477 \times 10^{-7}$ & $\mathrm{C} / \mathrm{T}$ & 0.078 & 0.012 & \\
\hline 2 & rs78658531 & 182741934 & G & 4942 & 6.925 & 3.232 & 14.84 & $6.477 \times 10^{-7}$ & $\mathrm{G} / \mathrm{A}$ & 0.078 & 0.012 & \\
\hline 2 & rs78797265 & 182736267 & $\mathrm{~T}$ & 4942 & 6.925 & 3.232 & 14.84 & $6.477 \times 10^{-7}$ & $\mathrm{~T} / \mathrm{G}$ & 0.078 & 0.012 & \\
\hline 2 & rs78890965 & 182734044 & $\mathrm{~T}$ & 4942 & 6.925 & 3.232 & 14.84 & $6.477 \times 10^{-7}$ & $\mathrm{~T} / \mathrm{C}$ & 0.078 & 0.012 & \\
\hline 10 & rs112889159 & 899303 & $\mathrm{~T}$ & 4942 & 8.161 & 3.564 & 18.69 & $6.807 \times 10^{-7}$ & $\mathrm{~T} / \mathrm{A}$ & 0.069 & 0.01 & LARP4B \\
\hline 8 & $8: 2410672$ & 2410672 & $\mathrm{~T}$ & 4942 & 7.257 & 3.318 & 15.88 & $6.952 \times 10^{-7}$ & $\mathrm{~T} / \mathrm{C}$ & 0.078 & 0.013 & \\
\hline 12 & rs116973965 & 34352942 & A & 4942 & 8.121 & 3.542 & 18.62 & $7.524 \times 10^{-7}$ & $\mathrm{~A} / \mathrm{G}$ & 0.069 & 0.011 & \\
\hline 2 & rs56272862 & 32379663 & $\mathrm{G}$ & 4942 & 3.995 & 2.307 & 6.917 & $7.610 \times 10^{-7}$ & $\mathrm{G} / \mathrm{A}$ & 0.157 & 0.045 & SPAST \\
\hline 17 & 17:77861401 & 77861401 & $\mathrm{~T}$ & 4942 & 7.375 & 3.328 & 16.34 & $8.604 \times 10^{-7}$ & $\mathrm{~T} / \mathrm{G}$ & 0.069 & 0.01 & \\
\hline 2 & rs72796871 & 32393157 & A & 4942 & 3.971 & 2.292 & 6.878 & $8.662 \times 10^{-7}$ & $\mathrm{~A} / \mathrm{G}$ & 0.157 & 0.046 & SLC30A6 \\
\hline 6 & rs1773013 & 2560712 & A & 4942 & 3.277 & 2.041 & 5.261 & $9.011 \times 10^{-7}$ & $\mathrm{~A} / \mathrm{G}$ & 0.245 & 0.092 & \\
\hline 2 & rs2303553 & 182783653 & $\mathrm{C}$ & 4942 & 6.723 & 3.141 & 14.39 & $9.218 \times 10^{-7}$ & $\mathrm{C} / \mathrm{T}$ & 0.078 & 0.012 & SSFA2 \\
\hline 2 & rs77278954 & 182793839 & A & 4942 & 6.723 & 3.141 & 14.39 & $9.218 \times 10^{-7}$ & $\mathrm{~A} / \mathrm{G}$ & 0.078 & 0.012 & SSFA2 \\
\hline 2 & rs78774163 & 182780126 & A & 4942 & 6.723 & 3.141 & 14.39 & $9.218 \times 10^{-7}$ & $\mathrm{~A} / \mathrm{G}$ & 0.078 & 0.012 & SSFA2 \\
\hline 8 & rs74463341 & 9228334 & $\mathrm{C}$ & 4942 & 7.027 & 3.219 & 15.34 & $9.852 \times 10^{-7}$ & $\mathrm{C} / \mathrm{G}$ & 0.078 & 0.014 & \\
\hline 2 & rs145475960 & 103130361 & A & 4942 & 5.499 & 2.778 & 10.89 & $9.995 \times 10^{-7}$ & $\mathrm{~A} / \mathrm{T}$ & 0.098 & 0.02 & SLC9A4 \\
\hline 2 & $2: 102820009$ & 102820009 & G & 4942 & 5.092 & 2.652 & 9.779 & $1.014 \times 10^{-6}$ & $\mathrm{G} / \mathrm{C}$ & 0.108 & 0.024 & IL1RL2 \\
\hline 2 & rs 13419200 & 182758257 & $\mathrm{C}$ & 4942 & 6.666 & 3.115 & 14.27 & $1.026 \times 10^{-6}$ & $\mathrm{C} / \mathrm{A}$ & 0.078 & 0.012 & SSFA2 \\
\hline 8 & rs74382792 & 62356700 & $\mathrm{G}$ & 4942 & 6.941 & 3.181 & 15.15 & $1.134 \times 10^{-6}$ & G/A & 0.078 & 0.014 & CLVS1 \\
\hline 17 & rs57769213 & 77879893 & G & 4942 & 7.199 & 3.251 & 15.94 & $1.138 \times 10^{-6}$ & $\mathrm{G} / \mathrm{C}$ & 0.069 & 0.011 & \\
\hline 20 & rs 140824800 & 12541106 & A & 4942 & 7.289 & 3.265 & 16.28 & $1.254 \times 10^{-6}$ & $\mathrm{~A} / \mathrm{G}$ & 0.069 & 0.011 & \\
\hline 12 & rs 146647050 & 38191129 & $\mathrm{~T}$ & 4942 & 5.931 & 2.871 & 12.25 & $1.514 \times 10^{-6}$ & $\mathrm{~T} / \mathrm{G}$ & 0.088 & 0.019 & \\
\hline 7 & rs 142711375 & 46602409 & G & 4942 & 6.503 & 3.028 & 13.97 & $1.581 \times 10^{-6}$ & $\mathrm{G} / \mathrm{A}$ & 0.078 & 0.014 & \\
\hline 5 & rs79287094 & 142892785 & G & 4942 & 8.409 & 3.522 & 20.08 & $1.624 \times 10^{-6}$ & G/A & 0.069 & 0.01 & \\
\hline 9 & rs 150057407 & 3276207 & G & 4942 & 4.92 & 2.563 & 9.445 & $1.672 \times 10^{-6}$ & $\mathrm{G} / \mathrm{T}$ & 0.108 & 0.024 & RFX3 \\
\hline 12 & rs143302148 & 39100013 & $\mathrm{~T}$ & 4942 & 7.507 & 3.286 & 17.15 & $1.739 \times 10^{-6}$ & $\mathrm{~T} / \mathrm{C}$ & 0.069 & 0.011 & CPNE8 \\
\hline 20 & rs76232775 & 60768910 & A & 4942 & 4.44 & 2.408 & 8.186 & $1.789 \times 10^{-6}$ & $\mathrm{~A} / \mathrm{G}$ & 0.118 & 0.03 & MTG2 \\
\hline 23 & rs 149305693 & 27808447 & $\mathrm{C}$ & 4942 & 8.11 & 3.435 & 19.15 & $1.799 \times 10^{-6}$ & $\mathrm{C} / \mathrm{T}$ & 0.069 & 0.012 & \\
\hline 9 & rs148123055 & 100176616 & G & 4942 & 6.626 & 3.044 & 14.42 & $1.886 \times 10^{-6}$ & G/A & 0.078 & 0.014 & TDRD7 \\
\hline 23 & rs1433806 & 27812073 & A & 4942 & 8.08 & 3.421 & 19.08 & $1.887 \times 10^{-6}$ & $\mathrm{~A} / \mathrm{G}$ & 0.069 & 0.011 & \\
\hline 12 & rs150862851 & 38793434 & $\mathrm{G}$ & 4942 & 7.435 & 3.255 & 16.98 & $1.928 \times 10^{-6}$ & $\mathrm{G} / \mathrm{A}$ & 0.069 & 0.012 & \\
\hline 23 & rs36115712 & 27825140 & A & 4942 & 8.066 & 3.415 & 19.05 & $1.931 \times 10^{-6}$ & $\mathrm{~A} / \mathrm{G}$ & 0.069 & 0.012 & \\
\hline 23 & rs 146644158 & 27819452 & $\mathrm{~T}$ & 4942 & 8.047 & 3.408 & 19 & $1.969 \times 10^{-6}$ & $\mathrm{~T} / \mathrm{C}$ & 0.069 & 0.012 & \\
\hline 23 & rs4829082 & 27805106 & $\mathrm{~T}$ & 4942 & 8.047 & 3.408 & 19 & $1.969 \times 10^{-6}$ & $\mathrm{~T} / \mathrm{C}$ & 0.069 & 0.012 & \\
\hline 23 & rs6630571 & 27814160 & A & 4942 & 8.047 & 3.408 & 19 & $1.969 \times 10^{-6}$ & $\mathrm{~A} / \mathrm{G}$ & 0.069 & 0.012 & \\
\hline 20 & rs149264569 & 49715107 & G & 4942 & 6.037 & 2.878 & 12.66 & $1.971 \times 10^{-6}$ & $\mathrm{G} / \mathrm{C}$ & 0.088 & 0.019 & \\
\hline 6 & rs9386997 & 111414038 & A & 4942 & 7.38 & 3.237 & 16.87 & $2.038 \times 10^{-6}$ & $\mathrm{~A} / \mathrm{T}$ & 0.069 & 0.011 & SLC16A10 \\
\hline 15 & rs62026663 & 45485831 & $\mathrm{C}$ & 4942 & 3.221 & 1.987 & 5.221 & $2.060 \times 10^{-6}$ & $\mathrm{C} / \mathrm{T}$ & 0.235 & 0.096 & SHF \\
\hline
\end{tabular}


Table 3 (continued)

\begin{tabular}{|c|c|c|c|c|c|c|c|c|c|c|c|c|}
\hline CHR & SNP & $\mathrm{BP}$ & Minor allele & $\mathrm{N}$ & OR & L95 & U95 & $p$ & GTPS & MAF cases & MAF controls & Gene \\
\hline 9 & rs 187960516 & 36238454 & A & 4942 & 7.132 & 3.164 & 16.07 & $2.155 \times 10^{-6}$ & $\mathrm{~A} / \mathrm{G}$ & 0.069 & 0.01 & CLTA-GNE \\
\hline 23 & rs140339686 & 27830115 & $\mathrm{~T}$ & 4942 & 7.963 & 3.372 & 18.81 & $2.226 \times 10^{-6}$ & $\mathrm{~T} / \mathrm{C}$ & 0.069 & 0.012 & \\
\hline 23 & rs4829084 & 27827112 & A & 4942 & 7.963 & 3.372 & 18.81 & $2.226 \times 10^{-6}$ & $\mathrm{~A} / \mathrm{G}$ & 0.069 & 0.012 & \\
\hline 6 & rs 73010912 & 155067310 & A & 4942 & 6.174 & 42.903 & 13.13 & $2.265 \times 10^{-6}$ & $\mathrm{~A} / \mathrm{G}$ & 0.088 & 0.015 & SCAF8 \\
\hline 6 & rs6921109 & 111448767 & $\mathrm{~T}$ & 4942 & 7.309 & 3.203 & 16.68 & $2.297 \times 10^{-6}$ & $\mathrm{~T} / \mathrm{A}$ & 0.069 & 0.011 & SLC16A10 \\
\hline 6 & rs7760668 & 111446502 & $\mathrm{C}$ & 4942 & 7.309 & 3.203 & 16.68 & $2.297 \times 10^{-6}$ & $\mathrm{C} / \mathrm{A}$ & 0.069 & 0.011 & SLC16A10 \\
\hline 23 & rs139460593 & 27817042 & $\mathrm{C}$ & 4942 & 7.925 & 3.357 & 18.71 & $2.319 \times 10^{-6}$ & $\mathrm{C} / \mathrm{T}$ & 0.069 & 0.012 & \\
\hline 6 & rs72993420 & 155087077 & $\mathrm{G}$ & 4942 & 6.129 & 2.882 & 13.03 & $2.475 \times 10^{-6}$ & G/A & 0.088 & 0.015 & SCAF8 \\
\hline 15 & rs62026667 & 45491136 & $\mathrm{G}$ & 4942 & 3.174 & 41.963 & 5.133 & $2.475 \times 10^{-6}$ & $\mathrm{G} / \mathrm{C}$ & 0.235 & 0.097 & SHF \\
\hline 15 & rs 142484525 & 95512720 & $\mathrm{~T}$ & 4942 & 6.364 & 42.944 & 13.76 & $2.535 \times 10^{-6}$ & $\mathrm{~T} / \mathrm{A}$ & 0.069 & 0.011 & \\
\hline
\end{tabular}

Top GWAS results based on 7,585,874 SNPs after imputation in 51 cases versus all 4891 population controls. All results were adjusted for genetic principal components $1-4$. The threshold for statistical significance was $p<5 \times 10^{-8}$

GWAS genome-wide association study, CHR chromosome, SNP single nucleotide polymorphism, BP base pair, $N$ number, GTPS Guanosine-5'triphosphates, $M A F$ minor allele frequency, $O R[95 \% C I]$ odds ratio with $95 \%$ confidence interval, $p p$ value

\section{Candidate Gene Analyses-Cases Versus Matched Controls}

When cases of bisphosphonate-associated AFF were compared with matched controls, no statistically significant associations were revealed (Fig. 2b; Table 6; Supplemental Table 2).

\section{Discussion}

We were hoping to find a strong common genetic susceptibility trait for AFF to predict patients at high risk of this ADR. Our results indicate that there is no common genetic variant that can be used for this purpose. The only significant finding on a genome-wide level was with four SNPs when cases were compared with population controls, but these were uncommon SNPs, all of which were single hits, meaning that these associations are likely false positives $[24,25]$, although two may theoretically be related to the treatment indication (NR3C1 and NTN1). None of these specific SNPs have, however, previously been implicated in AFF or osteoporosis [11, 26-28]. After reducing the risk of confounding by indication with the use of a comparison to bisphosphonate-treated controls, no statistically significant association remained.

At this time we are therefore left to models based on pharmacological and clinical considerations to minimize the risk of AFF. The prevailing pathophysiological theory of AFF is that bisphosphonates lead to over-suppression of bone remodeling [29]. Because bisphosphonates preferentially suppress the targeted repair mechanism, increased numbers of micro-cracks and reduced heterogeneity of the bone can be seen in bone tissue from animals and humans [7,
30-32]. The combination of these can lead to accumulation of micro-cracks during normal loading and propagation to larger cracks, eventually resulting in complete AFF. Studies have shown that the risk of developing an AFF is on average 50-fold greater for a bisphosphonate user compared to a nonuser, and more than 100-fold greater after 4-5 years of treatment $[3,5,33]$. In contrast, discontinuation of the drug will lead to a steep decline in the risk for developing an AFF [3]. In addition, different bisphosphonates might vary in terms of risk [3, 5, 34]. Hence, treatment duration and choice of bisphosphonate could be subject to manipulation in order to gain maximum treatment benefit while reducing the risk of AFF.

Many attempts have been made to identify risk factors that may predispose bisphosphonate users to AFF. A potential genetic influence has been suggested as a possible explanation to why only a minority of bisphosphonate users develop AFF. For instance, studies have revealed that polymorphisms in the gene encoding farnesyl diphosphate synthase (FDPS) may affect bone mineral density and bone turnover following bisphosphonate treatment in some patients, while not in others [35-38]. A possible genetic cause is also supported by studies that have demonstrated a difference in risk of AFF based on ethnicity, with Asians being at higher risk. A recent study by Lo et al. revealed a hazard ratio of 6.6 for females of Asian ethnicity compared with Caucasian women [9]. In addition, theories of a possible genetic trait have been long existing for other bisphosphonate ADRs that manifest in the skeleton [39].

There are several limitations to this study. First, matching of controls was done using bisphosphonate exposure as a proxy for osteoporosis as the Swedish Patient Register mainly includes information on diagnoses from hospital 
Table 4 Top genome-wide associations with bisphosphonate-associated atypical femoral fractures — cases vs matched controls

\begin{tabular}{|c|c|c|c|c|c|c|c|c|c|c|c|c|}
\hline CHR & SNP & $\mathrm{BP}$ & Minor allele & $\mathrm{N}$ & OR & L95 & U95 & $p$ & GTPS & MAF case & MAF control & Gene \\
\hline 16 & rs7188484 & 88918607 & $\mathrm{~T}$ & 375 & 3.576 & 2.153 & 5.94 & $8.605 \times 10^{-7}$ & $\mathrm{~T} / \mathrm{G}$ & 0.431 & 0.196 & GALNS \\
\hline 3 & rs6768500 & 2693258 & $\mathrm{C}$ & 375 & 7.634 & 3.379 & 17.25 & $1.021 \times 10^{-6}$ & $\mathrm{C} / \mathrm{G}$ & 0.157 & 0.023 & CNTN4 \\
\hline 3 & rs76646538 & 2694727 & $\mathrm{C}$ & 375 & 7.634 & 3.379 & 17.25 & $1.021 \times 10^{-6}$ & $\mathrm{C} / \mathrm{T}$ & 0.157 & 0.023 & CNTN4 \\
\hline 1 & rs1913592 & 18550837 & $\mathrm{C}$ & 375 & 3.346 & 2.06 & 5.435 & $1.055 \times 10^{-6}$ & $\mathrm{C} / \mathrm{T}$ & 0.529 & 0.279 & IGSF21 \\
\hline 12 & rs4765913 & 2419896 & A & 375 & 3.114 & 1.942 & 4.995 & $2.454 \times 10^{-6}$ & $\mathrm{~A} / \mathrm{T}$ & 0.412 & 0.188 & CACNA1C \\
\hline 16 & rs 12444242 & 88911043 & $\mathrm{~T}$ & 375 & 3.269 & 1.987 & 5.38 & $3.125 \times 10^{-6}$ & $\mathrm{~T} / \mathrm{C}$ & 0.402 & 0.182 & GALNS \\
\hline 16 & rs 12447646 & 88910824 & A & 375 & 3.269 & 1.987 & 5.38 & $3.125 \times 10^{-6}$ & $\mathrm{~A} / \mathrm{G}$ & 0.402 & 0.182 & GALNS \\
\hline 16 & rs 12449164 & 88909788 & $\mathrm{~T}$ & 375 & 3.269 & 1.987 & 5.38 & $3.125 \times 10^{-6}$ & $\mathrm{~T} / \mathrm{C}$ & 0.402 & 0.182 & GALNS \\
\hline 16 & rs8054592 & 88912039 & $\mathrm{~T}$ & 375 & 3.269 & 1.987 & 5.38 & $3.125 \times 10^{-6}$ & $\mathrm{~T} / \mathrm{C}$ & 0.402 & 0.182 & GALNS \\
\hline 16 & rs 12932521 & 88914235 & $\mathrm{~T}$ & 375 & 3.242 & 1.97 & 5.335 & $3.679 \times 10^{-6}$ & $\mathrm{~T} / \mathrm{C}$ & 0.402 & 0.184 & GALNS \\
\hline 16 & rs 34858110 & 88914598 & $\mathrm{C}$ & 375 & 3.242 & 1.97 & 5.335 & $3.679 \times 10^{-6}$ & $\mathrm{C} / \mathrm{A}$ & 0.402 & 0.184 & GALNS \\
\hline 16 & rs71395332 & 88909028 & $\mathrm{~T}$ & 375 & 3.243 & 1.97 & 5.336 & $3.683 \times 10^{-6}$ & $\mathrm{~T} / \mathrm{C}$ & 0.402 & 0.184 & GALNS \\
\hline 16 & rs 12598981 & 88916036 & $\mathrm{~T}$ & 375 & 3.217 & 1.955 & 5.293 & $4.278 \times 10^{-6}$ & $\mathrm{~T} / \mathrm{G}$ & 0.402 & 0.185 & GALNS \\
\hline 16 & rs11076726 & 88912899 & $\mathrm{~T}$ & 375 & 3.219 & 1.953 & 5.306 & $4.503 \times 10^{-6}$ & $\mathrm{~T} / \mathrm{G}$ & 0.422 & 0.201 & GALNS \\
\hline 8 & rs 17063092 & 3104832 & $\mathrm{C}$ & 375 & 2.958 & 1.86 & 4.703 & $4.614 \times 10^{-6}$ & $\mathrm{C} / \mathrm{T}$ & 0.461 & 0.238 & CSMD1 \\
\hline 7 & rs 12538221 & 24123003 & $\mathrm{~T}$ & 375 & 5.237 & 2.575 & 10.65 & $4.867 \times 10^{-6}$ & $\mathrm{~T} / \mathrm{C}$ & 0.167 & 0.045 & \\
\hline 7 & rs 71526045 & 24118952 & A & 375 & 5.237 & 2.575 & 10.65 & $4.867 \times 10^{-6}$ & $\mathrm{~A} / \mathrm{G}$ & 0.167 & 0.045 & \\
\hline 17 & rs61753147 & 8809025 & A & 375 & 5.265 & 2.58 & 10.74 & $4.995 \times 10^{-6}$ & $\mathrm{~A} / \mathrm{G}$ & 0.167 & 0.035 & PIK3R5 \\
\hline 16 & rs34495980 & 88906555 & A & 375 & 3.177 & 1.93 & 5.232 & $5.544 \times 10^{-6}$ & $\mathrm{~A} / \mathrm{C}$ & 0.402 & 0.188 & GALNS \\
\hline 15 & rs4776851 & 67180920 & A & 375 & 6.06 & 2.776 & 13.23 & $6.075 \times 10^{-6}$ & $\mathrm{~A} / \mathrm{G}$ & 0.137 & 0.031 & \\
\hline 16 & $16: 88906780$ & 88906780 & $\mathrm{G}$ & 375 & 3.152 & 1.914 & 5.191 & $6.427 \times 10^{-6}$ & G/A & 0.402 & 0.19 & GALNS \\
\hline 16 & rs 13337256 & 88907043 & $\mathrm{G}$ & 375 & 3.152 & 1.914 & 5.191 & $6.427 \times 10^{-6}$ & G/A & 0.402 & 0.19 & GALNS \\
\hline 16 & rs3784881 & 88905888 & $\mathrm{~T}$ & 375 & 3.152 & 1.914 & 5.191 & $6.427 \times 10^{-6}$ & $\mathrm{~T} / \mathrm{C}$ & 0.402 & 0.19 & GALNS \\
\hline 18 & rs116941264 & 75460371 & A & 375 & 10.78 & 3.833 & 30.33 & $6.609 \times 10^{-6}$ & $\mathrm{~A} / \mathrm{G}$ & 0.098 & 0.011 & \\
\hline 7 & rs2727797 & 36628761 & $\mathrm{~T}$ & 375 & 3.142 & 1.909 & 5.169 & $6.613 \times 10^{-6}$ & $\mathrm{~T} / \mathrm{C}$ & 0.676 & 0.44 & AOAH \\
\hline 10 & rs7082862 & 134341963 & $\mathrm{G}$ & 375 & 3.851 & 2.139 & 6.93 & $6.916 \times 10^{-6}$ & $\mathrm{G} / \mathrm{C}$ & 0.226 & 0.071 & \\
\hline 2 & rs 11465606 & 102988300 & A & 375 & 6.86 & 2.958 & 15.91 & $7.252 \times 10^{-6}$ & $\mathrm{~A} / \mathrm{C}$ & 0.128 & 0.022 & IL18R1 \\
\hline 8 & rs 17319624 & 3105800 & A & 375 & 3.161 & 1.906 & 5.242 & $8.180 \times 10^{-6}$ & $\mathrm{~A} / \mathrm{G}$ & 0.363 & 0.176 & CSMD1 \\
\hline 10 & rs 36009580 & 73627786 & G & 375 & 2.965 & 1.839 & 4.78 & $8.200 \times 10^{-6}$ & $\mathrm{G} / \mathrm{C}$ & 0.412 & 0.194 & \\
\hline 3 & rs2717296 & 182456980 & $\mathrm{C}$ & 375 & 2.864 & 1.802 & 4.552 & $8.603 \times 10^{-6}$ & $\mathrm{C} / \mathrm{T}$ & 0.686 & 0.426 & \\
\hline 8 & rs 17319596 & 3104594 & $\mathrm{C}$ & 375 & 2.846 & 1.795 & 4.513 & $8.681 \times 10^{-6}$ & $\mathrm{C} / \mathrm{T}$ & 0.461 & 0.245 & CSMD1 \\
\hline 8 & rs17319617 & 3105038 & A & 375 & 3.103 & 1.878 & 5.126 & $9.811 \times 10^{-6}$ & $\mathrm{~A} / \mathrm{C}$ & 0.363 & 0.176 & CSMD1 \\
\hline 8 & rs 34162586 & 3105087 & $\mathrm{C}$ & 375 & 3.103 & 1.878 & 5.126 & $9.811 \times 10^{-6}$ & $\mathrm{C} / \mathrm{G}$ & 0.363 & 0.176 & CSMD1 \\
\hline 8 & rs 35729878 & 3104896 & $\mathrm{G}$ & 375 & 3.103 & 1.878 & 5.126 & $9.811 \times 10^{-6}$ & $\mathrm{G} / \mathrm{C}$ & 0.363 & 0.176 & CSMD1 \\
\hline 15 & rs62026663 & 45485831 & $\mathrm{C}$ & 375 & 3.643 & 2.054 & 6.463 & $9.814 \times 10^{-6}$ & $\mathrm{C} / \mathrm{T}$ & 0.235 & 0.083 & SHF \\
\hline 8 & $8: 3104001$ & 3104001 & $\mathrm{~T}$ & 375 & 3.578 & 2.032 & 6.3 & $1.007 \times 10^{-5}$ & $\mathrm{~T} / \mathrm{G}$ & 0.245 & 0.096 & CSMD1 \\
\hline 8 & rs 117459261 & 3103995 & $\mathrm{~T}$ & 375 & 3.578 & 2.032 & 6.3 & $1.007 \times 10^{-5}$ & $\mathrm{~T} / \mathrm{A}$ & 0.245 & 0.096 & CSMD1 \\
\hline 8 & rs 73185574 & 3106144 & $\mathrm{C}$ & 375 & 3.099 & 1.874 & 5.124 & $1.041 \times 10^{-5}$ & $\mathrm{C} / \mathrm{T}$ & 0.363 & 0.179 & CSMD1 \\
\hline 8 & rs142418205 & 3097543 & G & 375 & 3.218 & 1.912 & 5.418 & $1.093 \times 10^{-5}$ & G/A & 0.343 & 0.167 & CSMD1 \\
\hline 16 & rs8062286 & 88917502 & A & 375 & 3.102 & 1.873 & 5.138 & $1.101 \times 10^{-5}$ & $\mathrm{~A} / \mathrm{G}$ & 0.402 & 0.198 & GALNS \\
\hline 7 & rs3801298 & 36569019 & $\mathrm{~T}$ & 375 & 3.098 & 1.87 & 5.131 & $1.123 \times 10^{-5}$ & $\mathrm{~T} / \mathrm{C}$ & 0.716 & 0.486 & AOAH \\
\hline 18 & rs3016811 & 589690 & $\mathrm{~T}$ & 375 & 2.609 & 1.7 & 4.002 & $1.126 \times 10^{-5}$ & $\mathrm{~T} / \mathrm{C}$ & 0.628 & 0.381 & \\
\hline 18 & rs518302 & 589635 & G & 375 & 2.609 & 1.7 & 4.002 & $1.126 \times 10^{-5}$ & G/A & 0.628 & 0.381 & \\
\hline 2 & rs6723676 & 22414978 & A & 375 & 2.821 & 1.774 & 4.484 & $1.159 \times 10^{-5}$ & $\mathrm{~A} / \mathrm{C}$ & 0.559 & 0.327 & \\
\hline 20 & rs 149264569 & 49715107 & $\mathrm{G}$ & 375 & 10.89 & 3.744 & 31.68 & $1.170 \times 10^{-5}$ & $\mathrm{G} / \mathrm{C}$ & 0.088 & 0.011 & \\
\hline 4 & rs116838635 & 112534842 & A & 375 & 5.704 & 2.617 & 12.43 & $1.187 \times 10^{-5}$ & $\mathrm{~A} / \mathrm{G}$ & 0.137 & 0.034 & \\
\hline 17 & rs111859148 & 32210110 & $\mathrm{C}$ & 375 & 3.174 & 1.893 & 5.323 & $1.191 \times 10^{-5}$ & $\mathrm{C} / \mathrm{T}$ & 0.304 & 0.13 & ASIC2 \\
\hline 17 & rs2348157 & 32210243 & G & 375 & 3.174 & 1.893 & 5.323 & $1.191 \times 10^{-5}$ & $\mathrm{G} / \mathrm{C}$ & 0.304 & 0.13 & ASIC2 \\
\hline 17 & rs56174865 & 32214269 & A & 375 & 3.174 & 1.893 & 5.323 & $1.191 \times 10^{-5}$ & $\mathrm{~A} / \mathrm{G}$ & 0.304 & 0.13 & ASIC2 \\
\hline 17 & rs66923090 & 32215593 & A & 375 & 3.174 & 1.893 & 5.323 & $1.191 \times 10^{-5}$ & $\mathrm{~A} / \mathrm{G}$ & 0.304 & 0.13 & ASIC2 \\
\hline
\end{tabular}


Table 4 (continued)

\begin{tabular}{lllllllllllll}
\hline CHR & SNP & BP & Minor allele & N & OR & L95 & U95 & $p$ & GTPS & MAF case & MAF control & Gene \\
\hline 17 & rs67026511 & 32215830 & G & 375 & 3.174 & 1.893 & 5.323 & $1.191 \times 10^{-5}$ & G/A & 0.304 & 0.13 & ASIC2 \\
17 & rs67236820 & 32215903 & A & 375 & 3.174 & 1.893 & 5.323 & $1.191 \times 10^{-5}$ & A/G & 0.304 & 0.13 & ASIC2 \\
17 & rs67809660 & 32215544 & $\mathrm{C}$ & 375 & 3.174 & 1.893 & 5.323 & $1.191 \times 10^{-5}$ & C/T & 0.304 & 0.13 & ASIC2 \\
17 & rs68033423 & 32215432 & $\mathrm{C}$ & 375 & 3.174 & 1.893 & 5.323 & $1.191 \times 10^{-5}$ & $\mathrm{C} / \mathrm{T}$ & 0.304 & 0.13 & ASIC2 \\
17 & rs68085213 & 32215389 & $\mathrm{C}$ & 375 & 3.1741 .893 & 5.323 & $1.191 \times 10^{-5}$ & $\mathrm{C} / \mathrm{T}$ & 0.304 & 0.13 & ASIC2 \\
17 & rs72818938 & 32215882 & $\mathrm{C}$ & 375 & 3.1741 .893 & 5.323 & $1.191 \times 10^{-5}$ & $\mathrm{C} / \mathrm{T}$ & 0.304 & 0.13 & ASIC2 \\
17 & rs8069564 & 32215953 & $\mathrm{~T}$ & 375 & 3.1741 .893 & 5.323 & $1.191 \times 10^{-5}$ & T/C & 0.304 & 0.13 & ASIC2 \\
17 & rs8070346 & 32212347 & $\mathrm{C}$ & 375 & 3.1741 .893 & 5.323 & $1.191 \times 10^{-5}$ & $\mathrm{C} / \mathrm{G}$ & 0.304 & 0.13 & ASIC2 \\
17 & rs8074055 & 32215922 & $\mathrm{C}$ & 375 & 3.1741 .893 & 5.323 & $1.191 \times 10^{-5}$ & $\mathrm{C} / \mathrm{T}$ & 0.304 & 0.13 & ASIC2 \\
17 & rs8076707 & 32212839 & $\mathrm{C}$ & 375 & 3.1741 .893 & 5.323 & $1.191 \times 10^{-5}$ & $\mathrm{C} / \mathrm{T}$ & 0.304 & 0.13 & ASIC2 \\
\hline
\end{tabular}

Top GWAS results based on 7,585,874 SNPs after imputation in 51 cases versus 324 matched controls. All results were adjusted for genetic principal components $1-4$. The threshold for statistical significance was $p<5 \times 10^{-8}$

GWAS genome-wide association study, CHR chromosome, SNP single nucleotide polymorphism, BP base pair, $N$ number, GTPS Guanosine-5'triphosphates, $M A F$ minor allele frequency, $O R[95 \% C I]$ odds ratio with $95 \%$ confidence interval, $p p$ value

care. We were thus unable to identify controls who were prescribed a bisphosphonate for osteoporosis prevention. Secondly, although this is the largest genetic study of bisphosphonate-associated AFF to date, the number of included cases is still low. This means that the power to detect weakly associated common variants and strongly associated rare variants is low. It is also possible that several variants, inherited independently of one another, are required to infer a risk of AFF, in which case they will go undetected. To elucidate this would require a larger study and whole genome or exome sequencing, which was beyond the scope of this study. Lastly, there are suggestions that the association between bisphosphonate use and AFF is mainly driven by a genetic predisposition [11]. However, since 4-5 years of bisphosphonate use in Swedish women is associated with a 125-fold increase in risk of AFF [3], the potential underlying causal genetic risk allele(-s) should have a firm relation with both AFF and bisphosphonate use to entirely extenuate the exponential increase in risk with duration of bisphosphonate use. Noteworthily, a more moderately strong effect modification between bisphosphonates and genetic predisposition might still exist, but the current study is too small to disentangle such genetic modifying effects.

That several genetic loci, perhaps varying between individuals, might explain at least some cases of bisphosphonate-associated AFF has been proposed by some studies, although methodological issues and other limitations makes it difficult to conclude whether the findings are of relevance for a larger population of individuals with bisphosphonate-associated AFF. In the study by PérezNúñez et al. that compared 13 women with AFF and 268 female controls, 21 loci were more frequent in the fracture group [40]. Most patients accumulated two or more allelic variants, and the number of variants was different between patients with fractures and the controls, suggesting that several genes may be involved. The study was, however, limited by the fact that the controls were a mix of normal and osteoporotic women, and that only 12 of the 13 cases had been exposed to bisphosphonates. In another study, Roca-Ayats et al. performed whole-exome sequencing in three sisters who had all developed AFF following bisphosphonate treatment, and compared with three unrelated patients with bisphosphonate-associated AFF [41]. They detected 37 rare nonsynonymous mutations in 34 genes, but the results are questionable due to lack of validation and a small sample size. In a further study, Funck-Brentano et al. performed sequencing of four genes amongst two patients with bisphosphonate-associated AFF and found genetic variants in one, a rare heterozygous mutation in COL1A2 (c.213G > A; p.Arg708GIn) [42]. Limitations of this study include the small sample size. While these findings suggest a polygenic model in which an accumulation of susceptibility variants may lead to a predisposition to bisphosphonate-associated AFF, larger studies are required to provide solid evidence.

\section{Conclusion}

With this genome-wide association and candidate gene study, we were unable to find evidence of common genetic traits predisposition for bisphosphonate-associated AFF. This does not rule out the possibility of weakly associated genetic traits or the presence of rare genetic variants that confer a risk. Further studies of larger sample size as well as whole-exome or whole-genome sequencing studies are warranted. 
A

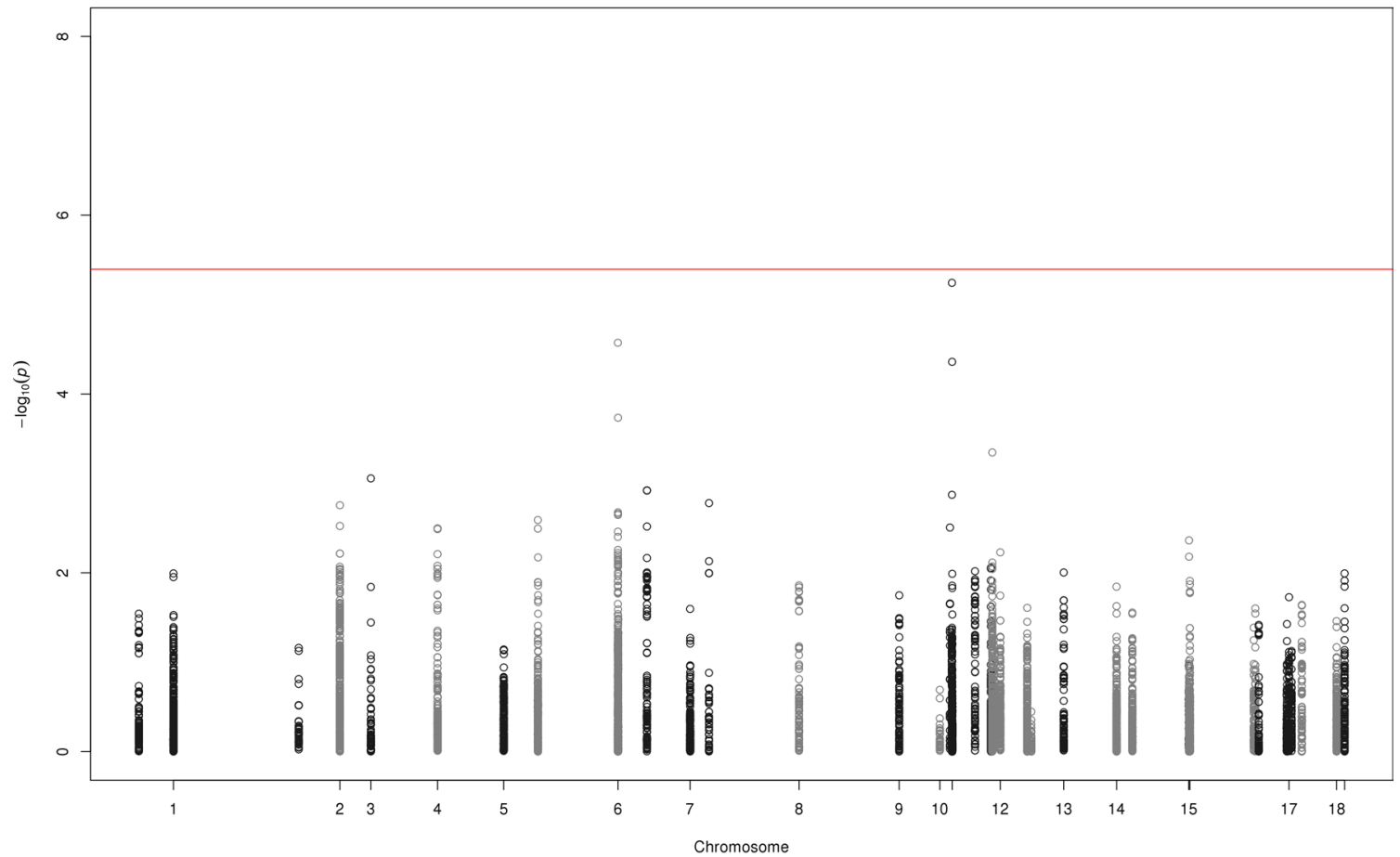

B

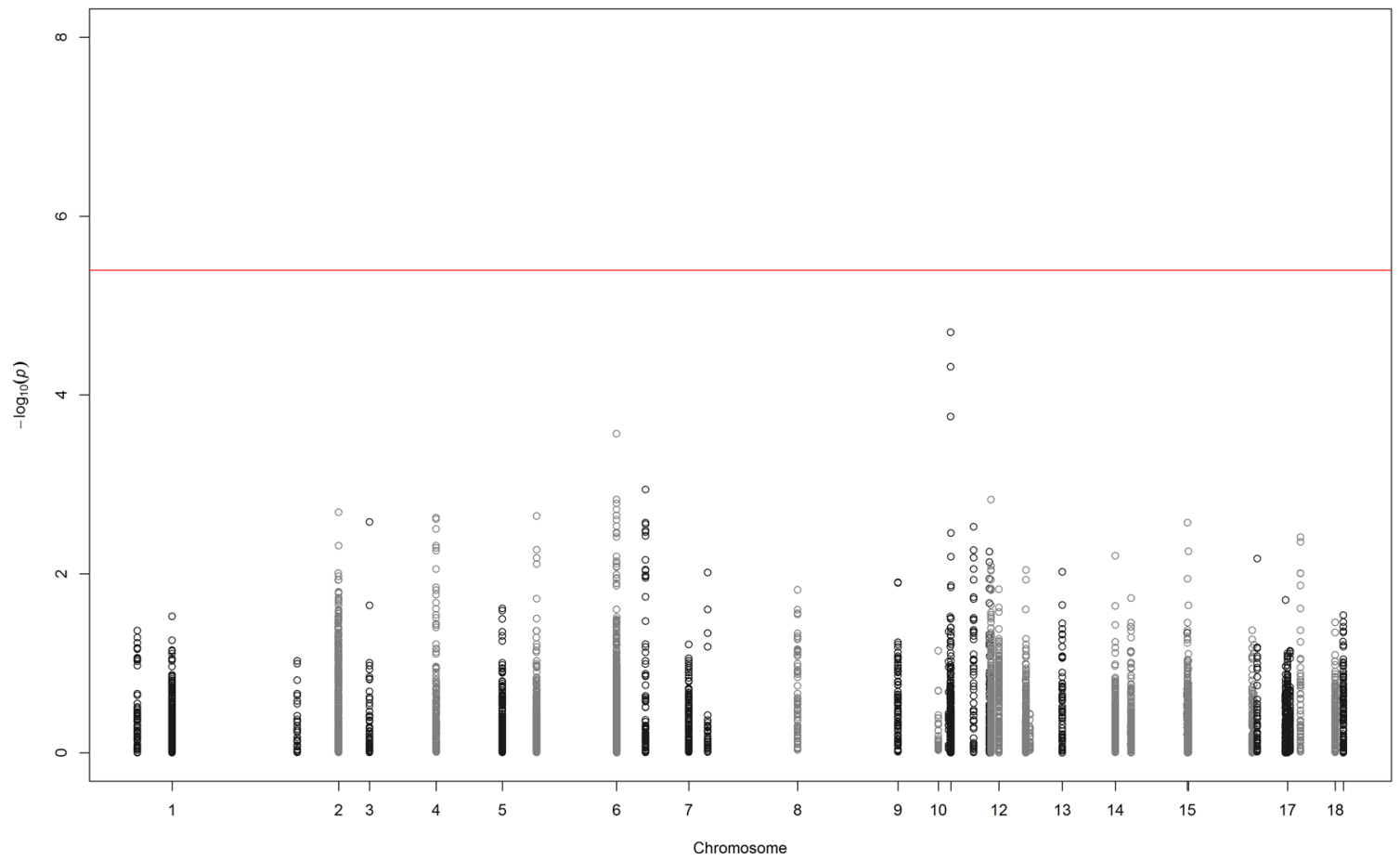

Fig. 2 a Manhattan plot of the candidate gene analyses - cases vs all 4891 controls. b Manhattan plot of the candidate gene analysescases vs matched controls. Analyses of 51 cases of bisphosphonateassociated atypical femoral fractures versus a all 4891 controls, and b 324 matched controls. Adjustment was made for genetic principal components $1-4$. The red line shows the threshold for statistical significance of $5.74 \times 10^{-6}$. There were no statistically significant associations in either analysis 


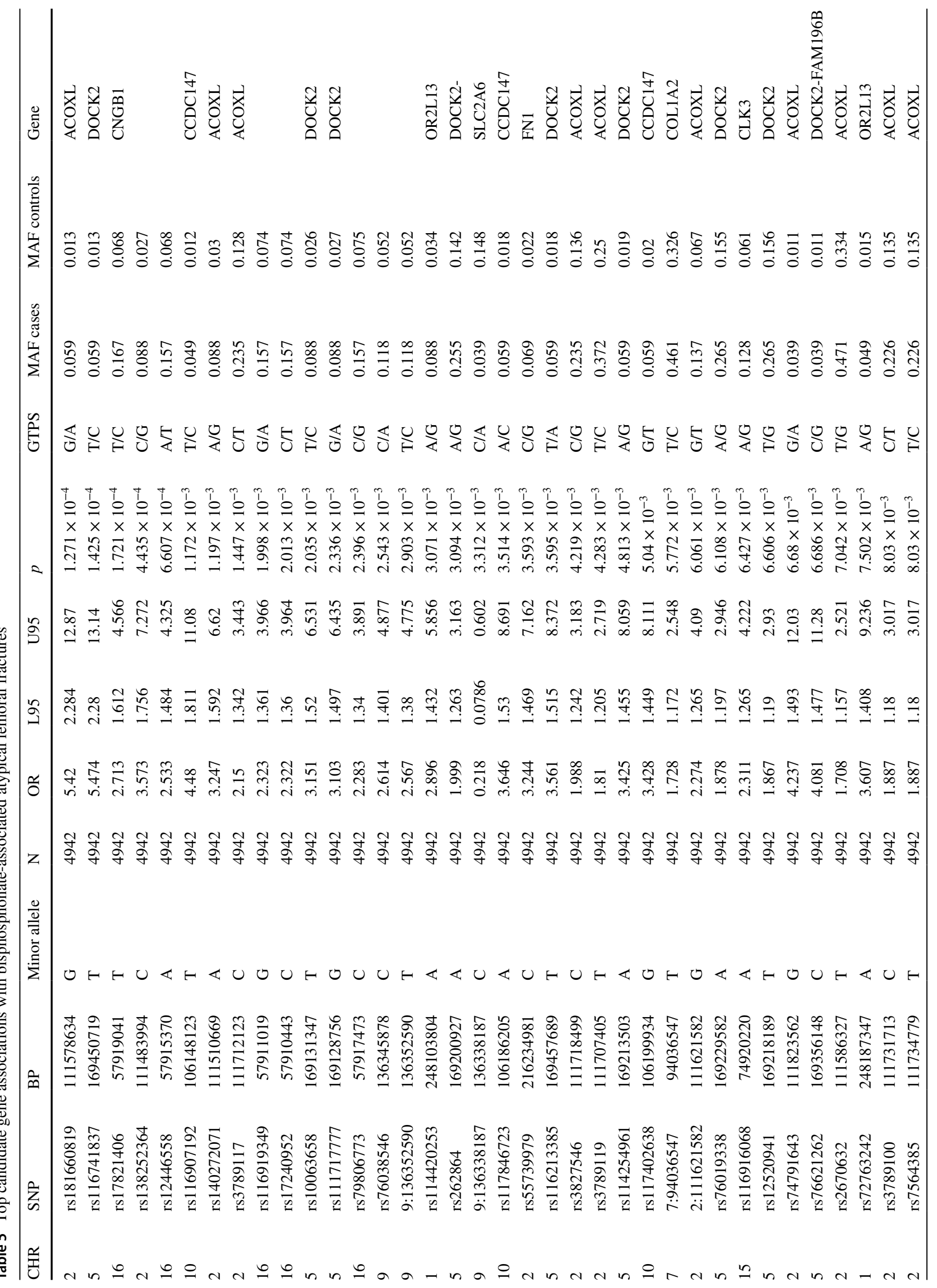




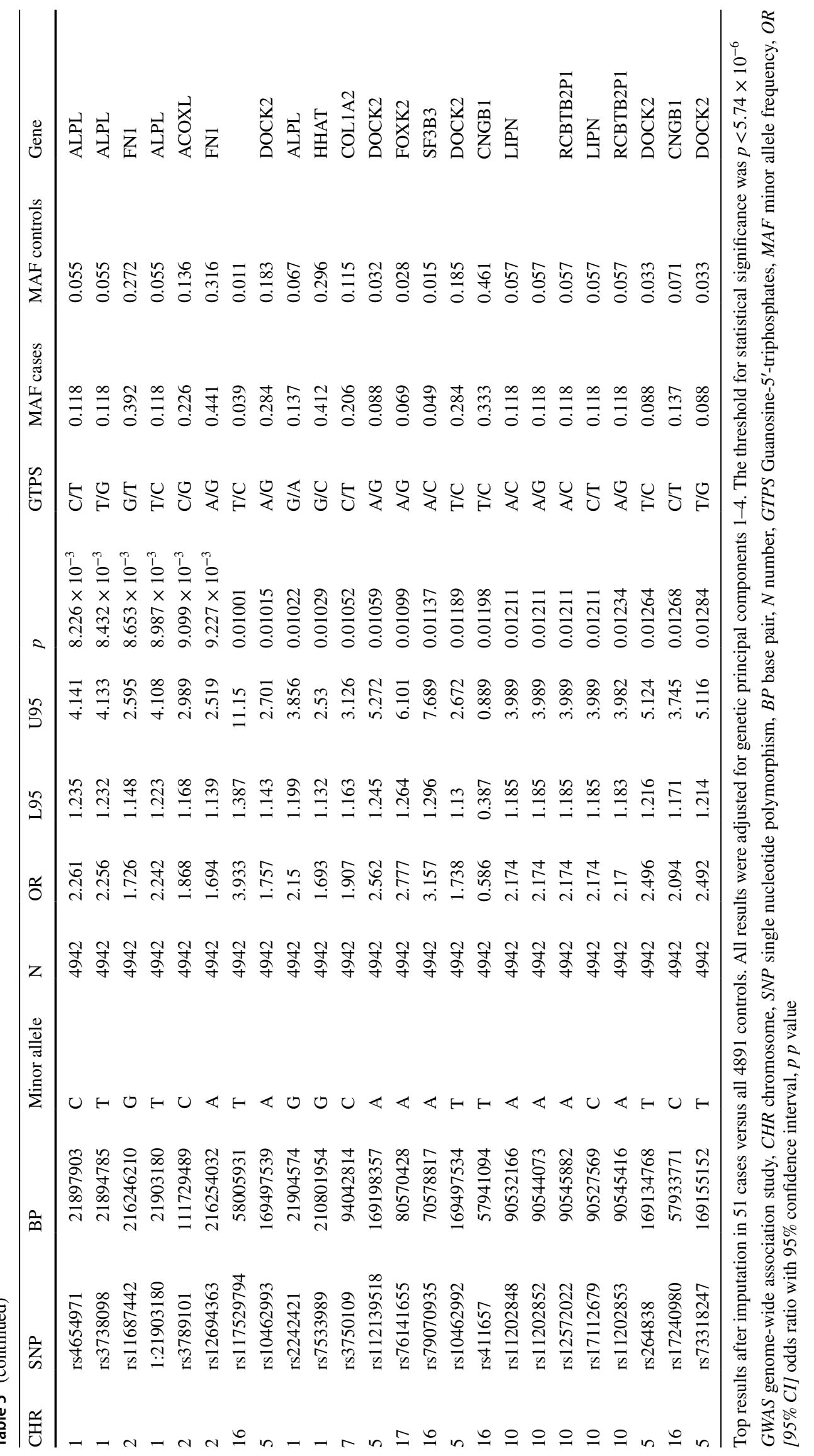




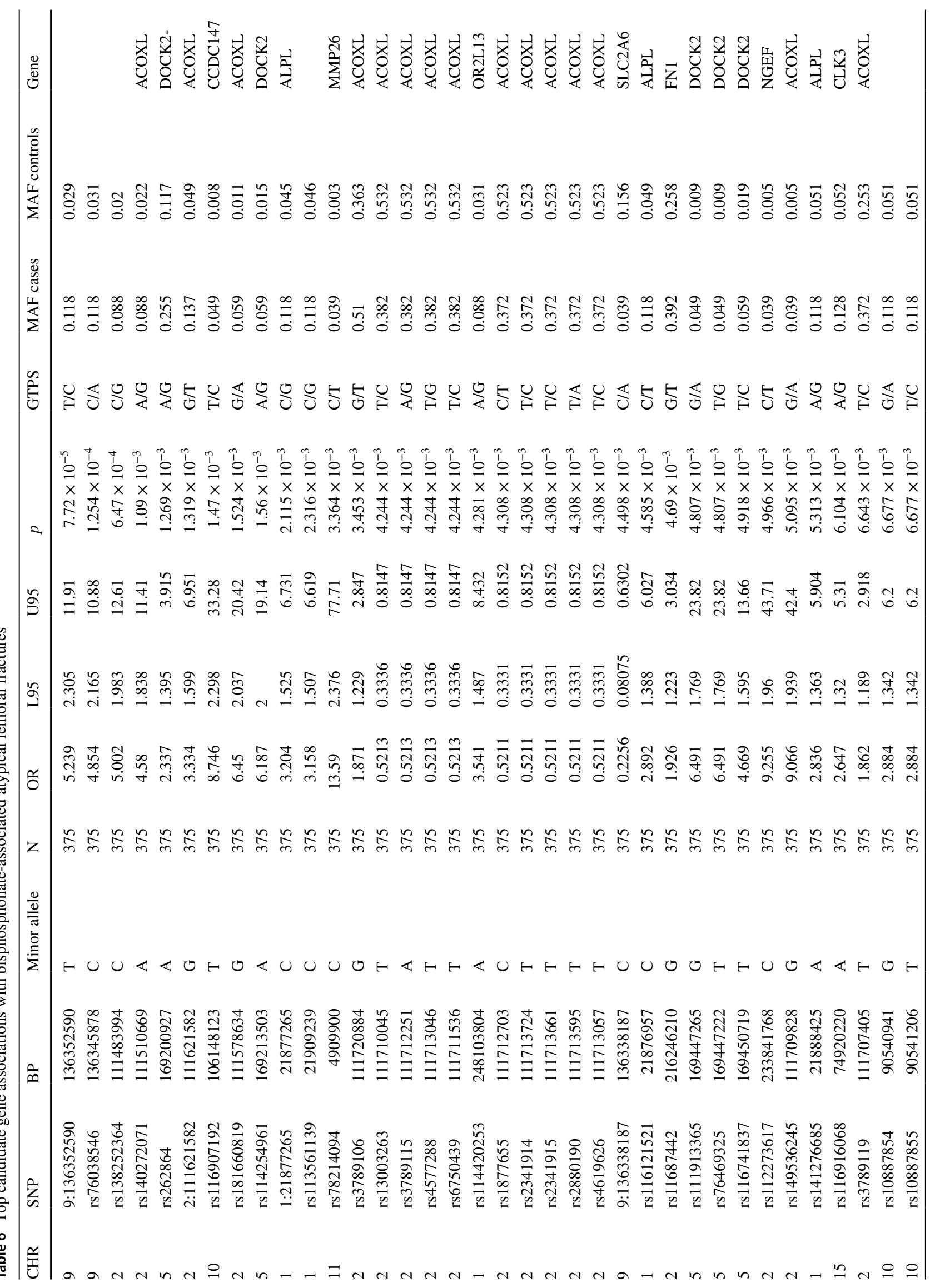




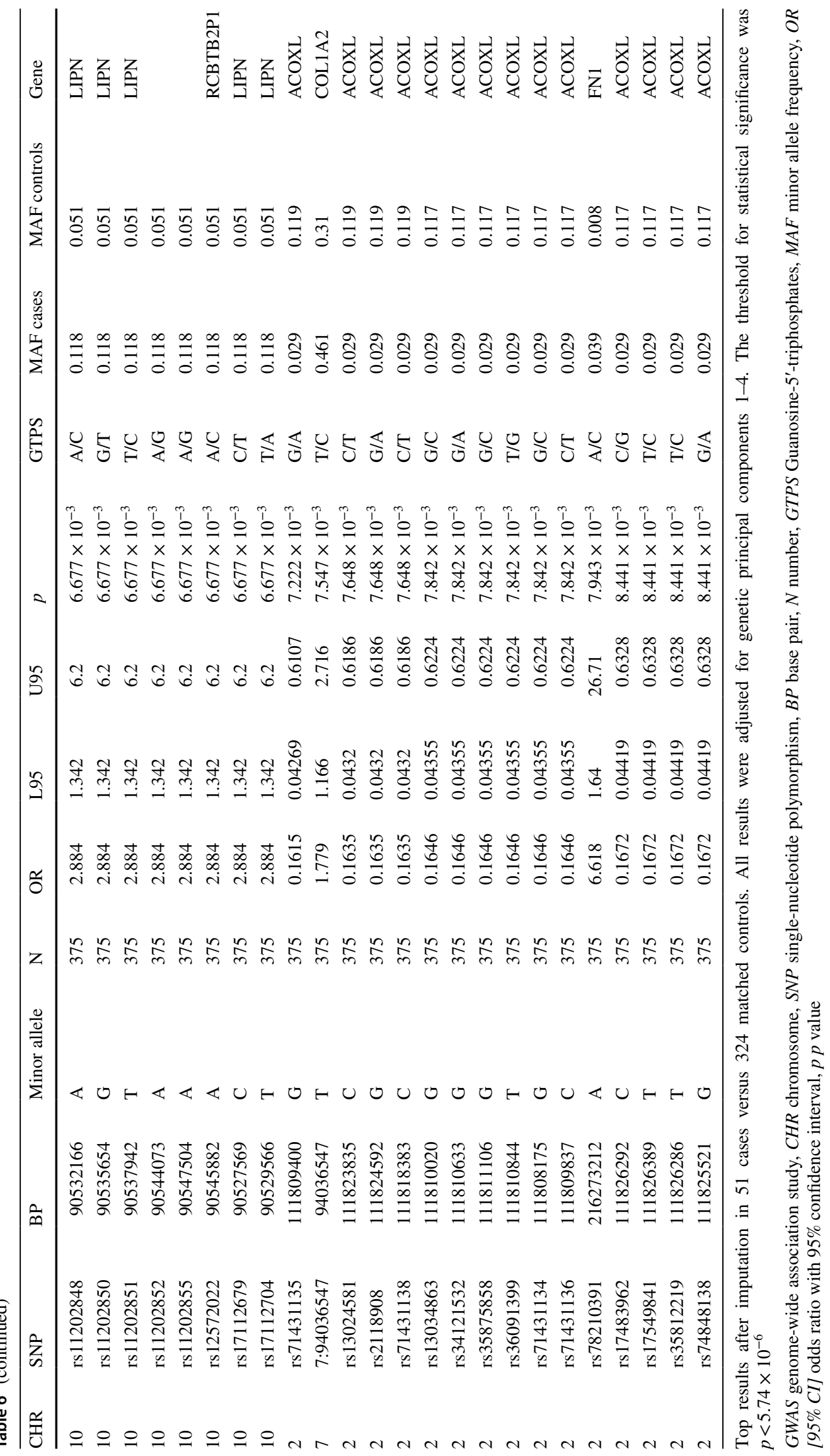


Acknowledgements This work was supported by the Swedish Research Council (Medicine 521-2011-2440, 521-2014-3370 and 2015-03527); Swedish Heart and Lung Foundation (20120557, 20140291 and 20170711); Selander's foundation; Thuréus' foundation; the Swedish Medical Products Agency; the Clinical Research Support (ALF) at Uppsala University; and Östergötland County Council (LIO-698411). We thank research nurses Ulrica Ramqvist, Elisabeth Stjernberg, Charlotta Haglund and Elisabeth Balcom, and research assistants Sofie Collin, Eva Prado Lopez, Agnes Kataja Knight, Agnes Wadelius, and Martha Wadelius, Department of Medical Sciences, Clinical Pharmacology, Uppsala University, Uppsala, Sweden, for recruiting and interviewing cases and for database administration. We are grateful to Tomas Axelsson for SNP array genotyping at the Department of Medical Sciences, SNP\&SEQ Technology Platform, which is funded by the Science for Life Laboratory, Swedish Research Council, and Uppsala University. Computations were performed on resources provided by SNIC through Uppsala Multidisciplinary Center for Advanced Computational Science (UPPMAX). We acknowledge Patrik Magnusson and Barbro Sandin at the Department of Medical Epidemiology and Biostatistics, Karolinska Institutet for access to data from the Swedish Twin Registry, which is managed by Karolinska Institutet and receives funding through the Research Council Swedish under Grant No. 2017-00641.

Author Contributions Study design: PH and MW. Data collection: MK, MW, KM, JS and PH. Data analysis: NE. Data interpretation: MK, PH, MW, KM, JS, HM and NE. Drafting manuscript: MK and PH. Revising manuscript content: MK, PH, MW, KM, JS, HM and NE. Approving final version of manuscript: MK, PH, MW, KM, JS, HM and NE.

\section{Compliance with Ethical Standards}

Conflict of interest Mohammad Kharazmi, Karl Michaëlsson, Jörg Schilcher, Niclas Eriksson, Håkan Melhus, Mia Wadelius, and Pär Hallberg declare that they have no conflict of interest.

Ethical Approval The study was approved by the regional ethical review boards in Uppsala and Stockholm (2010/231 in Uppsala; 2007/644-31 and 2011/463-32 in Stockholm).

Informed Consent Written informed consent was obtained from all participants.

Open Access This article is distributed under the terms of the Creative Commons Attribution 4.0 International License (http://creativeco mmons.org/licenses/by/4.0/), which permits unrestricted use, distribution, and reproduction in any medium, provided you give appropriate credit to the original author(s) and the source, provide a link to the Creative Commons license, and indicate if changes were made.

\section{References}

1. Adler RA (2018) Management of endocrine disease: atypical femoral fractures: risks and benefits of long-term treatment of osteoporosis with anti-resorptive therapy. Eur J Endocrinol 178:R81-R87

2. Shane E, Burr D, Abrahamsen B, Adler RA, Brown TD, Cheung AM, Cosman F, Curtis JR, Dell R, Dempster DW, Ebeling PR, Einhorn TA, Genant HK, Geusens P, Klaushofer K, Lane JM, McKiernan F, McKinney R, Ng A, Nieves J, O'Keefe R, Papapoulos S, Howe TS, van der Meulen MC, Weinstein RS, Whyte MP (2014) Atypical subtrochanteric and diaphyseal femoral fractures: second report of a task force of the American Society for Bone and Mineral Research. J Bone Miner Res 29:1-23

3. Schilcher J, Koeppen V, Aspenberg P, Michaelsson K (2014) Risk of atypical femoral fracture during and after bisphosphonate use. N Engl J Med 371:974-976

4. Schilcher J, Michaelsson K, Aspenberg P (2011) Bisphosphonate use and atypical fractures of the femoral shaft. N Engl J Med 364:1728-1737

5. Meier RP, Perneger TV, Stern R, Rizzoli R, Peter RE (2012) Increasing occurrence of atypical femoral fractures associated with bisphosphonate use. Arch Intern Med 172:930-936

6. Khosla S, Cauley JA, Compston J, Kiel DP, Rosen C, Saag KG, Shane E (2017) Addressing the crisis in the treatment of osteoporosis: a path forward. J Bone Miner Res 32(3):424-430

7. Aspenberg P, Schilcher J (2014) Atypical femoral fractures, bisphosphonates, and mechanical stress. Curr Osteoporos Rep 12:189-193

8. Kharazmi M, Hallberg P, Michaelsson K (2014) Gender related difference in the risk of bisphosphonate associated atypical femoral fracture and osteonecrosis of the jaw. Ann Rheum Dis 73:1594

9. Lo JC, Hui RL, Grimsrud CD, Chandra M, Neugebauer RS, Gonzalez JR, Budayr A, Lau G, Ettinger B (2016) The association of race/ethnicity and risk of atypical femur fracture among older women receiving oral bisphosphonate therapy. Bone 85:142-147

10. Schilcher J (2015) High revision rate but good healing capacity of atypical femoral fractures. A comparison with common shaft fractures. Injury 46:2468-2473

11. Nguyen HH, van der Laarschot DM, Verkerk AJ, Milat F, Zillikens MC, Ebeling PR (2018) Genetic risk factors for atypical femoral fractures (AFFs): a systematic review. J Bone Miner Res Plus 2:2-12

12. Carr DF, Pirmohamed M (2018) Biomarkers of adverse drug reactions. Exp Biol Med (Maywood) 243:291-299

13. Magnusson PK, Almqvist C, Rahman I, Ganna A, Viktorin A, Walum H, Halldner L, Lundstrom S, Ullen F, Langstrom N, Larsson H, Nyman A, Gumpert CH, Rastam M, Anckarsater H, Cnattingius S, Johannesson M, Ingelsson E, Klareskog L, de Faire U, Pedersen NL, Lichtenstein P (2013) The Swedish Twin Registry: establishment of a biobank and other recent developments. Twin Res Hum Genet 16:317-329

14. Chang CC, Chow CC, Tellier LC, Vattikuti S, Purcell SM, Lee JJ (2015) Second-generation PLINK: rising to the challenge of larger and richer datasets. Gigascience 4:7

15. McCarthy S, Das S, Kretzschmar W, Delaneau O, Wood AR, Teumer A, Kang HM, Fuchsberger C, Danecek P, Sharp K, Luo Y, Sidore C, Kwong A, Timpson N, Koskinen S, Vrieze S, Scott LJ, Zhang H, Mahajan A, Veldink J, Peters U, Pato C, van Duijn CM, Gillies CE, Gandin I, Mezzavilla M, Gilly A, Cocca M, Traglia M, Angius A, Barrett JC, Boomsma D, Branham K, Breen G, Brummett CM, Busonero F, Campbell H, Chan A, Chen S, Chew E, Collins FS, Corbin LJ, Smith GD, Dedoussis G, Dorr M, Farmaki AE, Ferrucci L, Forer L, Fraser RM, Gabriel S, Levy S, Groop L, Harrison T, Hattersley A, Holmen OL, Hveem K, Kretzler M, Lee JC, McGue M, Meitinger T, Melzer D, Min JL, Mohlke KL, Vincent JB, Nauck M, Nickerson D, Palotie A, Pato M, Pirastu N, McInnis M, Richards JB, Sala C, Salomaa V, Schlessinger D, Schoenherr S, Slagboom PE, Small K, Spector T, Stambolian D, Tuke M, Tuomilehto J, Van den Berg LH, Van Rheenen W, Volker U, Wijmenga C, Toniolo D, Zeggini E, Gasparini P, Sampson MG, Wilson JF, Frayling T, de Bakker PI, Swertz MA, McCarroll S, Kooperberg C, Dekker A, Altshuler D, Willer C, Iacono W, Ripatti S et al (2016) A reference panel of 64,976 haplotypes for genotype imputation. Nat Genet 48:1279-1283 
16. Loh PR, Danecek P, Palamara PF, Fuchsberger C, Reshef YA, Finucane HK, Schoenherr S, Forer L, McCarthy S, Abecasis GR, Durbin R (2016) Reference-based phasing using the Haplotype Reference Consortium panel. Nat Genet 48:1443-1448

17. Durbin R (2014) Efficient haplotype matching and storage using the positional Burrows-Wheeler transform (PBWT). Bioinformatics 30:1266-1272

18. Sham PC, Purcell SM (2014) Statistical power and significance testing in large-scale genetic studies. Nat Rev Genet 15:335-346

19. Purcell S, Cherny SS, Sham PC (2003) Genetic power calculator: design of linkage and association genetic mapping studies of complex traits. Bioinformatics 19:149-150

20. GeneCards In:Weizmann Institute of Science

21. Ragnarsson O, Glad CA, Bergthorsdottir R, Almqvist EG, Ekerstad E, Widell H, Wangberg B, Johannsson G (2015) Body composition and bone mineral density in women with Cushing's syndrome in remission and the association with common genetic variants influencing glucocorticoid sensitivity. Eur J Endocrinol 172:1-10

22. Szappanos A, Patocs A, Toke J, Boyle B, Sereg M, Majnik J, Borgulya G, Varga I, Liko I, Racz K, Toth M (2009) BclI polymorphism of the glucocorticoid receptor gene is associated with decreased bone mineral density in patients with endogenous hypercortisolism. Clin Endocrinol (Oxf) 71:636-643

23. Morris JA, Kemp JP, Youlten SE, Laurent L, Logan JG, Chai RC, Vulpescu NA, Forgetta V, Kleinman A, Mohanty ST, Sergio CM, Quinn J, Nguyen-Yamamoto L, Luco AL, Vijay J, Simon MM, Pramatarova A, Medina-Gomez C, Trajanoska K, Ghirardello EJ, Butterfield NC, Curry KF, Leitch VD, Sparkes PC, Adoum AT, Mannan NS, Komla-Ebri DSK, Pollard AS, Dewhurst HF, Hassall TAD, Beltejar MG, Me Research T, Adams DJ, Vaillancourt SM, Kaptoge S, Baldock P, Cooper C, Reeve J, Ntzani EE, Evangelou E, Ohlsson C, Karasik D, Rivadeneira F, Kiel DP, Tobias JH, Gregson CL, Harvey NC, Grundberg E, Goltzman D, Adams DJ, Lelliott CJ, Hinds DA, Ackert-Bicknell CL, Hsu YH, Maurano MT, Croucher PI, Williams GR, Bassett JHD, Evans DM, Richards JB (2019) An atlas of genetic influences on osteoporosis in humans and mice. Nat Genet 51:258-266

24. McRae AF (2017) Analysis of genome-wide association data. Methods Mol Biol 1526:161-173

25. Teo YY (2008) Common statistical issues in genome-wide association studies: a review on power, data quality control, genotype calling and population structure. Curr Opin Lipidol 19:133-143

26. Estrada K, Styrkarsdottir U, Evangelou E, Hsu YH, Duncan EL, Ntzani EE, Oei L, Albagha OM, Amin N, Kemp JP, Koller DL, Li G, Liu CT, Minster RL, Moayyeri A, Vandenput L, Willner D, Xiao SM, Yerges-Armstrong LM, Zheng HF, Alonso N, Eriksson J, Kammerer CM, Kaptoge SK, Leo PJ, Thorleifsson G, Wilson SG, Wilson JF, Aalto V, Alen M, Aragaki AK, Aspelund T, Center JR, Dailiana Z, Duggan DJ, Garcia M, Garcia-Giralt N, Giroux S, Hallmans G, Hocking LJ, Husted LB, Jameson KA, Khusainova R, Kim GS, Kooperberg C, Koromila T, Kruk M, Laaksonen M, Lacroix AZ, Lee SH, Leung PC, Lewis JR, Masi L, Mencej-Bedrac S, Nguyen TV, Nogues X, Patel MS, Prezelj J, Rose LM, Scollen S, Siggeirsdottir K, Smith AV, Svensson O, Trompet S, Trummer O, van Schoor NM, Woo J, Zhu K, Balcells S, Brandi ML, Buckley BM, Cheng S, Christiansen C, Cooper C, Dedoussis G, Ford I, Frost M, Goltzman D, Gonzalez-Macias J, Kahonen M, Karlsson M, Khusnutdinova E, Koh JM, Kollia P, Langdahl BL, Leslie WD, Lips P, Ljunggren O, Lorenc RS, Marc J, Mellstrom D, Obermayer-Pietsch B, Olmos JM, Pettersson-Kymmer U, Reid DM, Riancho JA, Ridker PM, Rousseau F, Slagboom PE, Tang NL et al (2012) Genome-wide meta-analysis identifies 56 bone mineral density loci and reveals 14 loci associated with risk of fracture. Nat Genet 44:491-501
27. Karasik D, Rivadeneira F, Johnson ML (2016) The genetics of bone mass and susceptibility to bone diseases. Nat Rev Rheumatol $12: 496$

28. Rivadeneira F, Makitie O (2016) Osteoporosis and bone mass disorders: from gene pathways to treatments. Trends Endocrinol Metab 27:262-281

29. Odvina CV, Zerwekh JE, Rao DS, Maalouf N, Gottschalk FA, Pak CY (2005) Severely suppressed bone turnover: a potential complication of alendronate therapy. J Clin Endocrinol Metab 90:1294-1301

30. Donnelly E, Meredith DS, Nguyen JT, Gladnick BP, Rebolledo BJ, Shaffer AD, Lorich DG, Lane JM, Boskey AL (2012) Reduced cortical bone compositional heterogeneity with bisphosphonate treatment in postmenopausal women with intertrochanteric and subtrochanteric fractures. J Bone Miner Res 27:672-678

31. Allen MR, Burr DB (2011) Bisphosphonate effects on bone turnover, microdamage, and mechanical properties: what we think we know and what we know that we don't know. Bone 49:56-65

32. Iwata K, Mashiba T, Hitora T, Yamagami Y, Yamamoto T (2014) A large amount of microdamages in the cortical bone around fracture site in a patient of atypical femoral fracture after long-term bisphosphonate therapy. Bone 64:183-186

33. Dell RM, Adams AL, Greene DF, Funahashi TT, Silverman SL, Eisemon EO, Zhou H, Burchette RJ, Ott SM (2012) Incidence of atypical nontraumatic diaphyseal fractures of the femur. J Bone Miner Res 27:2544-2550

34. Kharazmi M, Hallberg P, Warfvinge G, Michaelsson K (2014) Risk of atypical femoral fractures and osteonecrosis of the jaw associated with alendronate use compared with other oral bisphosphonates. Rheumatology (Oxford) 53:1911-1913

35. Marini F, Falchetti A, Silvestri S, Bagger Y, Luzi E, Tanini A, Christiansen C, Brandi ML (2008) Modulatory effect of farnesyl pyrophosphate synthase (FDPS) rs2297480 polymorphism on the response to long-term amino-bisphosphonate treatment in postmenopausal osteoporosis. Curr Med Res Opin 24:2609-2615

36. Olmos JM, Zarrabeitia MT, Hernandez JL, Sanudo C, GonzalezMacias J, Riancho JA (2012) Common allelic variants of the farnesyl diphosphate synthase gene influence the response of osteoporotic women to bisphosphonates. Pharmacogenomics J $12: 227-232$

37. Choi HJ, Choi JY, Cho SW, Kang D, Han KO, Kim SW, Kim SY, Chung YS, Shin CS (2010) Genetic polymorphism of geranylgeranyl diphosphate synthase (GGSP1) predicts bone density response to bisphosphonate therapy in Korean women. Yonsei Med J 51:231-238

38. Liu Y, Liu H, Li M, Zhou P, Xing X, Xia W, Zhang Z, Liao E, Chen D, Liu J, Tao T, Wu W, Xu L (2014) Association of farnesyl diphosphate synthase polymorphisms and response to alendronate treatment in Chinese postmenopausal women with osteoporosis. Chin Med J (Engl) 127:662-668

39. Sarasquete ME, Garcia-Sanz R, Marin L, Alcoceba M, Chillon MC, Balanzategui A, Santamaria C, Rosinol L, de la Rubia J, Hernandez MT, Garcia-Navarro I, Lahuerta JJ, Gonzalez M, San Miguel JF (2008) Bisphosphonate-related osteonecrosis of the jaw is associated with polymorphisms of the cytochrome P450 CYP2C8 in multiple myeloma: a genome-wide single nucleotide polymorphism analysis. Blood 112:2709-2712

40. Perez-Nunez I, Perez-Castrillon JL, Zarrabeitia MT, Garcia-Ibarbia C, Martinez-Calvo L, Olmos JM, Briongos LS, Riancho J, Camarero V, Munoz Vives JM, Cruz R, Riancho JA (2015) Exon array analysis reveals genetic heterogeneity in atypical femoral fractures. A pilot study. Mol Cell Biochem 409:45-50 
41. Roca-Ayats N, Balcells S, Garcia-Giralt N, Falco-Mascaro M, Martinez-Gil N, Abril JF, Urreizti R, Dopazo J, Quesada-Gomez JM, Nogues X, Mellibovsky L, Prieto-Alhambra D, Dunford JE, Javaid MK, Russell RG, Grinberg D, Diez-Perez A (2017) GGPS1 mutation and atypical femoral fractures with bisphosphonates. $\mathrm{N}$ Engl J Med 376:1794-1795
42. Funck-Brentano T, Ostertag A, Debiais F, Fardellone P, Collet C, Mornet E, Cohen-Solal M (2017) Identification of a p.Arg708Gln variant in COL1A2 in atypical femoral fractures. Joint Bone Spine 84:715-718

Publisher's Note Springer Nature remains neutral with regard to jurisdictional claims in published maps and institutional affiliations. 